\title{
ANÁLISIS DE VISIBILIDAD SATÉLITAL Y TIEMPO DE VIAJE EN CADA CENTRO REGIONAL DE LA RED GEODÉSICA ACTIVA DE LA UNAH EN EL AÑO 2018
}

\author{
Antonio Carías ${ }^{1, *}$, Manuel Rodríguez ${ }^{2,+}$ Yeny Castellanos ${ }^{1,+}$ \\ Yessica Sosa ${ }^{1, \S}$, Marcela Norori ${ }^{1, \mathbb{I}}$ \\ ${ }^{1}$ Departamento de Ciencia y Técnologías de la Información Geográfica, UNAH \\ ${ }^{2}$ Departamento de Física de la Tierra, UNAH
}

Recibido: 09/agosto/2019

Aceptado: 08/noviembre/2019

DOI: https://doi.org/10.5377/ce.v12i1.9627

\begin{abstract}
RESUMEN
El uso del sistema GPS contribuye en el mejoramiento de la posición, con la utilización de las estaciones de operación continua podemos llegar a altas precisiones con un mantenimiento de bajo costo, pero aun así todos estos elementos deben de ser analizados para que la red sea óptima. Este estudio busca identificar el tipo de observación GPS que tiene cada centro regional en donde están ubicados los receptores de medición continua GPS, los obstáculos que puede tener la antena ya instalada y la propia disponibilidad de satélites en la región hondureña. Así mismo la realización de un análisis de desplazamiento con relación tiempo-terreno el cual sirve para la planificación de las giras de supervisión-mantenimiento con el objetivo de que dichas giras sean rentables.
\end{abstract}

Palabras clave: GPS, acceso, visibilidad satelital.

\begin{abstract}
The use of the GPS system contributes to the improvement of the position, with the use of continuous operation stations we can reach high precision with low-cost maintenance, but still all these elements must be analyzed so that the network is optimal. This study seeks to identify the type of GPS observation that each regional center has where the GPS continuous measurement receivers are located, the obstacles that the antenna may already have installed and the availability of satellites in the Honduran region. Likewise, the realization of a displacement analysis with a time-terrain relationship which serves to plan the supervision-maintenance tours so that these tours are profitable.
\end{abstract}

Keywords: GPS, acces, satellite visibility.

\footnotetext{
*antonio.carias@unah.edu.hn

${ }^{+}$manuelr@uwalumni.com

‡ycastellanos@unah.edu.hn

\$yessica.sosa@unah.edu.hn

Ijohana.norori@unah.edu.hn
} 


\section{Introducción}

El avance de la tecnología de la navegación por satélite es indudable, pero el tipo de precisión depende del tipo de receptor en este caso el geodésico multifrecuencia, el cual puede llegar a precisiones milimétricas. El mercado masivo de estos aparatos ha provocado un descenso en el precio de estos (GNSS Agency, 2013).

El mercado GPS en la actualidad ha tenido diferentes técnicas de diferenciación para tratar de mejorar la precisión y mitigar el error. El beneficio de la mejora del rendimiento de los receptores GPS de bajo costo ha llevado a su uso en aplicaciones tales como monitoreo de deslizamientos, tectónica de placas, trabajos de topografía, trabajos precisos con drones etc.

Teniendo una red de estaciones de operación continua GPS instaladas en los centros regionales de la Universidad Nacional Autónoma de Honduras es importante tener claro los aspectos de visibilidad de cada centro regional para así tener buena disponibilidad de los satélites, así mismo con los altos costos de movilización en el país, es importante conocer el costo promedio en tiempo de desplazamiento a cada centro regional para los respectivos mantenimientos que se puedan requerir en los diferentes equipos.

Para tener teóricamente claro el alcance del estudio revisaremos brevemente algunos de los conceptos que se analizaron:

\subsection{Visibilidad satélital}

El funcionamiento adecuado de un receptor GPS requiere la recepción ininterrumpida de la señal de al menos cuatro satélites GPS. Sin embargo, las señales de ondas de radio GPS no pueden penetrar bosques o casas en muchos casos, este empobrecimiento de la señal será temporal y, por lo tanto, no obstaculizará gravemente la posición cuando estemos en zonas rurales, pero con estructuras artificiales, como paredes, presas, edificios y puentes en zonas urbanas del interior de la ciudad con edificios muy altos, la visibilidad de los satélites GPS a menudo está limitada por períodos prolongados o simplemente no está disponible, a este error se le llama error de multitrayectoria.

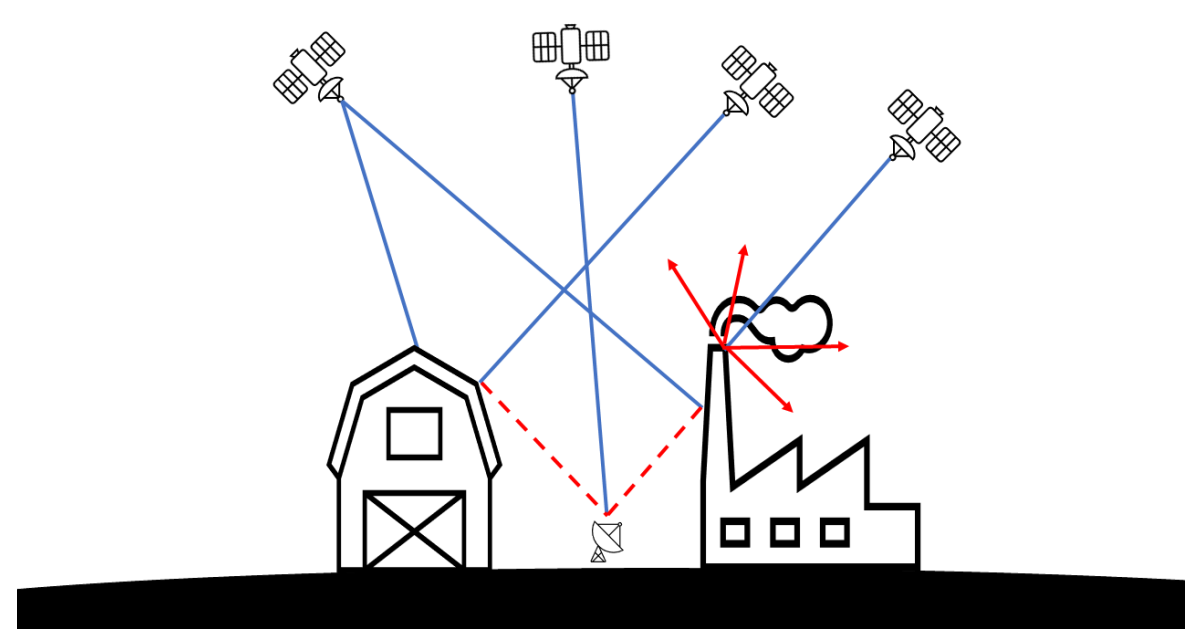

Figura 1: Error multitrayectoria. 
Otro error importante de tener en cuenta en la visibilidad satelital es la geometría satelital, ya que esta cambia durante el tiempo debido al movimiento relativo en la órbita de los satélites. La distribución de los satélites sobre el horizonte de un observador influye directamente en la calidad de la posición derivada de ellos. Como algunos de sus precursores, la precisión de una posición GPS está sujeta a un fenómeno geométrico llamado dilución de precisión (DOP). Este número es algo similar a la fortaleza de la consideración de la figura en el diseño de una red de triangulación. DOP se refiere a la resistencia geométrica de la figura descrita por las posiciones de los satélites entre sí y los receptores GPS.

Un bajo factor DOP es bueno; un alto factor DOP es malo. En otras palabras, cuando los satélites están en la configuración óptima para una posición GPS confiable, el DOP es bajo; cuando no lo están, el DOP es alto (ver figura 2 y figura 3 ).

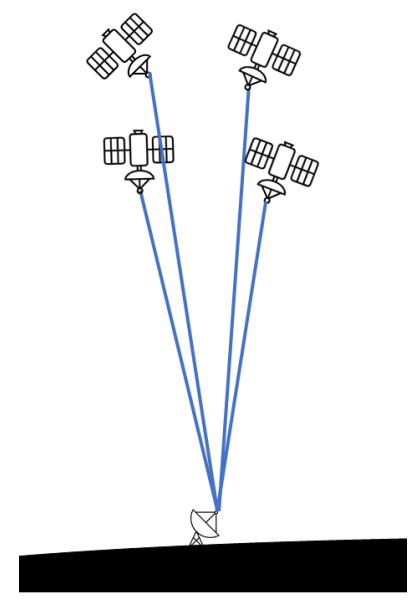

Figura 2: Mal PDOP

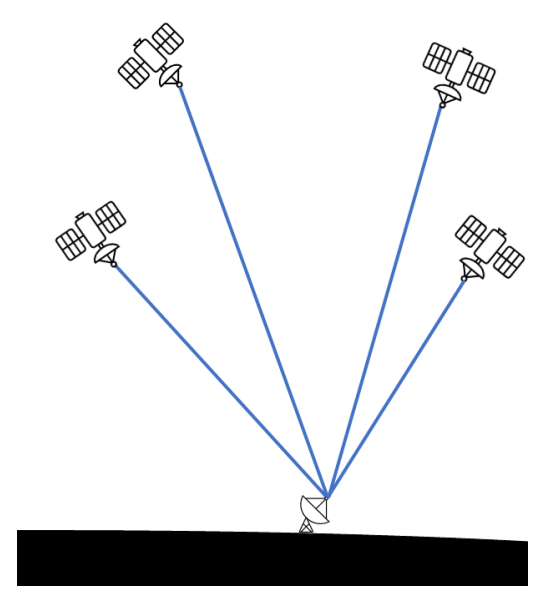

Figura 3: Buen PDOP

La premisa inicial para adquirir una coordenada es que al menos cuatro satélites deben de ser observables por encima de la máscara configurada en el receptor, sin embargo, si todos estos satélites están apiñados en una parte del cielo, es probable que la posición tenga una incertidumbre inaceptable y que el DOP, o dilución de precisión, sea alto. En otras palabras, un DOP alto es como una advertencia de que los errores reales en una posición GPS pueden ser más grandes de lo que podría esperar (Sickle, 2015).

\subsection{Tiempo de viaje}

Recientemente, la fiabilidad del tiempo de viaje se ha convertido en un factor muy importante para evaluar las condiciones del tráfico de una carretera determinada. El personal técnico espera contar con tiempos de viaje estables y confiables. Por lo tanto, la confiabilidad del tiempo de viaje puede considerarse como un factor importante en la evaluación de las condiciones del tráfico de la ruta elegida (Lee et al., 2019).

La congestión y el estado de las carreteras es uno de los problemas más notorios a la hora de planificar la rentabilidad de un viaje por carretera. Debido a estos problemas, los conductores están tratando de encontrar las rutas más cortas y confiables para llegar al sitio destino. En cuanto a la fiabilidad del tiempo de viaje, ya existen algunos métodos con Sistemas de Información Geográfica (SIG) los cuales demuestran el tiempo de viaje con respecto a la vialidad y los diferentes accesos para llegar a los sitios destinos. Por lo tanto, la confiabilidad del tiempo de viaje se ha convertido en un importante criterio de desempeño para las giras de supervisión y mantenimiento, complementando las medidas tradicionales, como el retraso y 
el tiempo promedio de viaje. En una investigación reciente, se han propuesto una variedad de medidas de rendimiento para cuantificar la fiabilidad y monetizarla. Esto incluye el tiempo de planificación, el tiempo de búfer, la desviación estándar, el coeficiente de variación, la asimetría, etc. (Lomax et al., 2003).

\section{Metodología}

\subsection{Disponibilidad del sistema GPS}

El buen funcionamiento del sistema GPS es limitado, y depende mucho de la visibilidad, de la conexión satélite-receptor, la latitud de las regiones, la geometría satélital, el almanaque recibido de las estaciones de referencia etc.

Comenzando con la latitud de la región en análisis, Honduras al estar cerca de la zona ecuatorial la cual tiene buena disponibilidad del sistema GPS. A excepción de las zonas polares en donde hay mucho problema debido a que los satélites no orbitan por esas zonas.

Las antenas que están instaladas en los centros regionales pertenecen a la marca Ashtech tipo choke ring modelo 701945 las cuales reducen el ruido o efecto multi-trayectoria, ver figura 4 . Y en la figura 5 se visualiza la ubicación de cada una de las estaciones permanentes ubicada en los Centros Regionales a nivel nacional

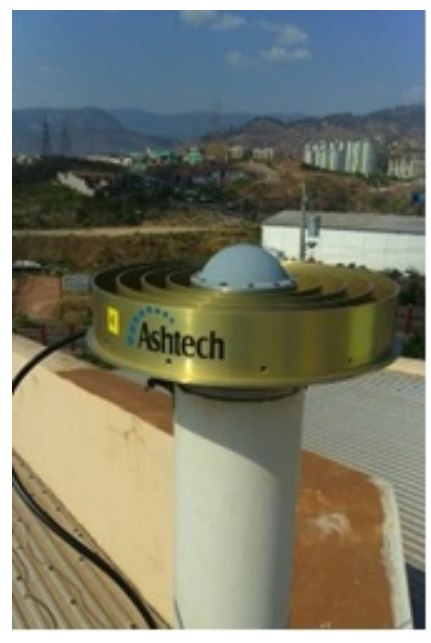

Figura 4: Antena GPS.

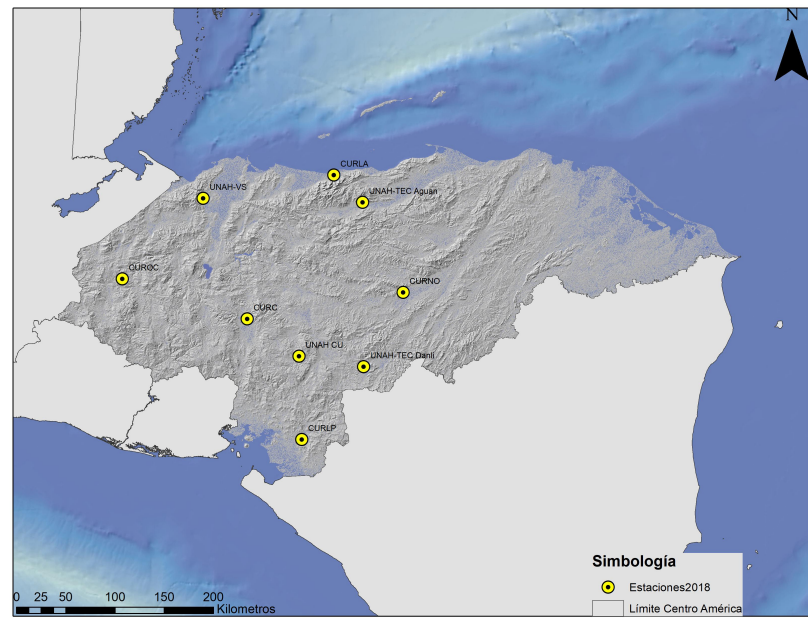

Figura 5: Ubicación de las estaciones permanentes.

En la observación realizada en el periodo del año 2018 para los diferentes centros regionales veremos que la disponibilidad es $24 / 7$ y con una disponibilidad promedio de 12 satélites observables, así como se puede ver en la figura 6 y la figura 7, obtenidas del sitio http://www.gnssmissionplanning.com/. 


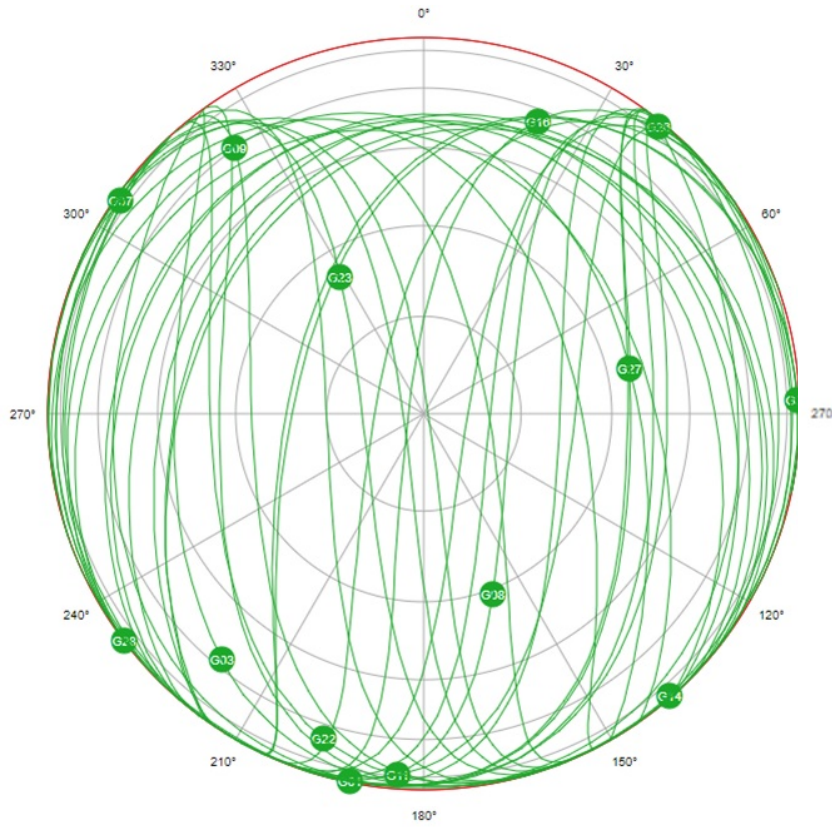

Figura 6: CURC.

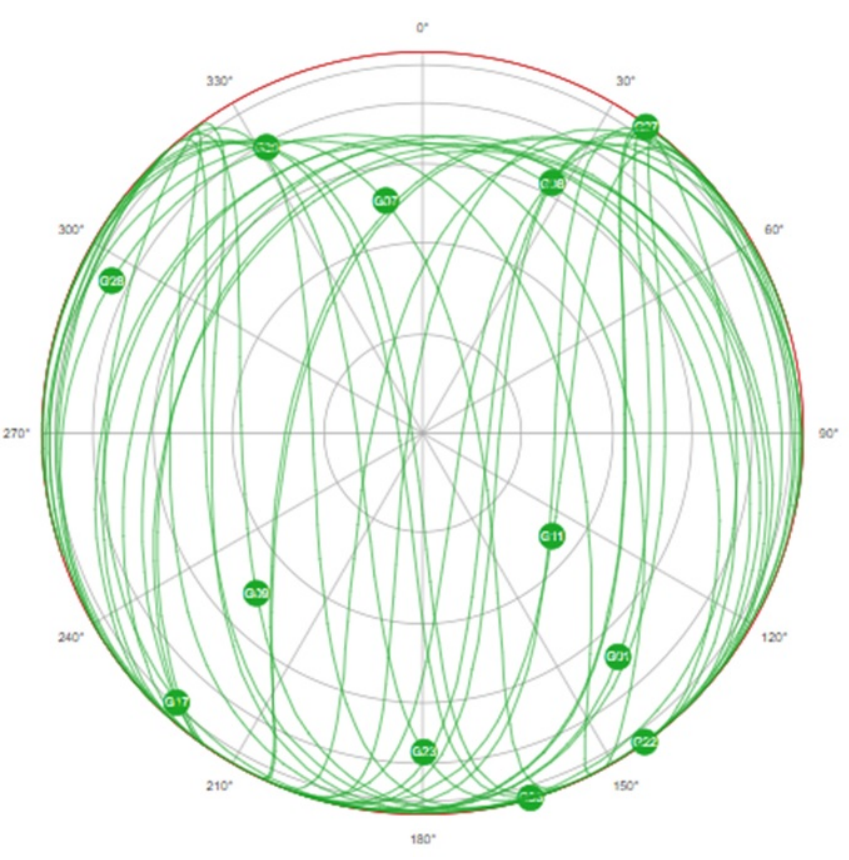

Figura 7: CURLA

La cobertura del cielo local varía en función de la latitud de la estación (Yahya y Kamarudin, 2008). Se observa que el problema en la visibilidad del satélite es más probable que se detecte, hacia la parte norte, donde casi no hay cobertura satélital. Al representar la configuración más deseable para el posicionamiento GPS, la cobertura local de GPS en los centros regionales está bien distribuida en todos los cuadrantes. En la figura 8 podremos observar los satélites observables en un periodo de 24 horas.

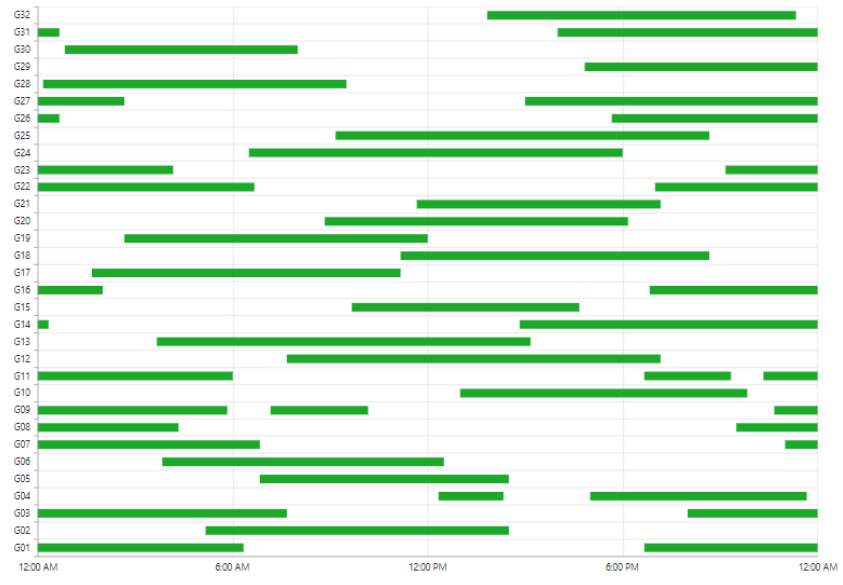

Figura 8: Observación satélital en 24 horas.

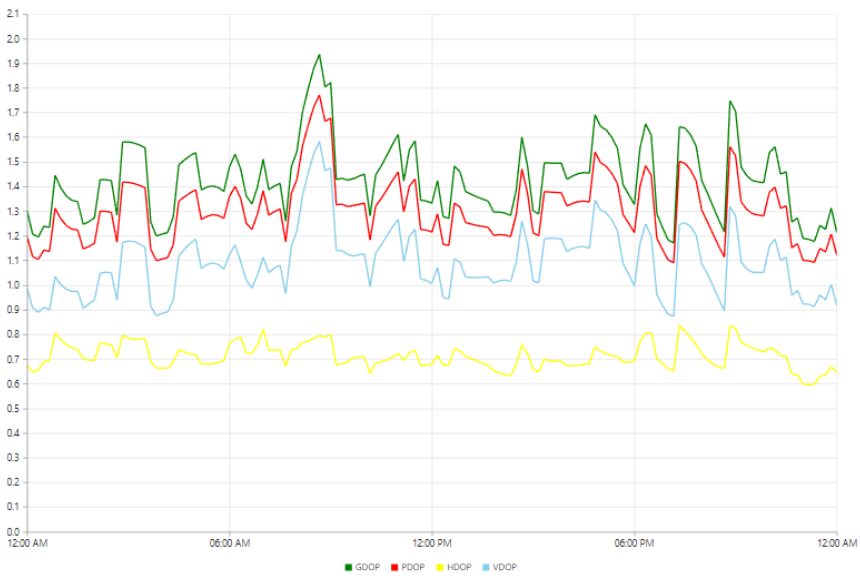

Figura 9: Valores DOP

Los valores de la figura 9 observados para el año 2018 presentan un buen valor en el HDOP que es la incertidumbre en la posición horizontal la cual da mayor precisión cuando menor es este valor, se considera que un valor por debajo de 4 es bueno, por lo tanto, todas las estaciones instaladas en los centros regionales están con valores muy buenos.

Se realizo un análisis de detección de obstáculos a nivel del suelo utilizando la metodología de línea vista calculada con una máscara de $15^{\circ}$ la se refleja en la figura 10 . 


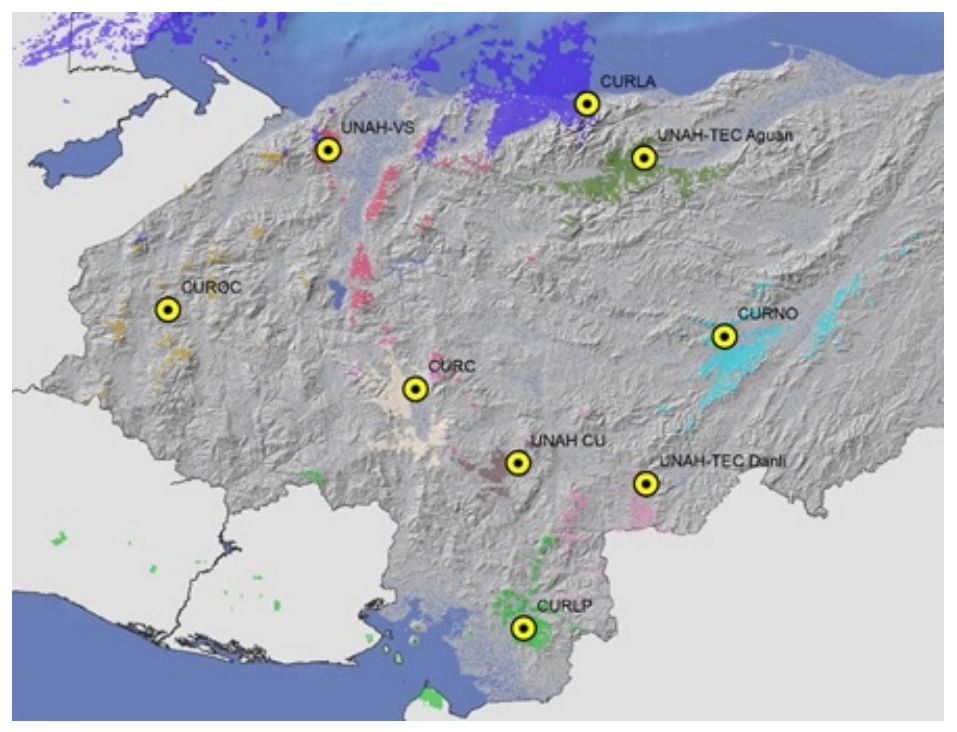

Figura 10: Análisis de visibilidad con máscara de $15^{\circ}$ en cada centro Regional de la UNAH. Fuente: Antonio Carías

Como se muestra en la figura 10 es claro que los lugares en donde hay mucha montaña hay que elevar el ángulo de la máscara para mejorar la incertidumbre de la posición (DOP) asumiendo que la propagación de una señal libre de interrupciones debido a errores multi-trayectoria sean mitigados por el receptor GPS, en este caso está demostrado que estamos dentro de un buen margen de DOP.

\subsection{Análisis del tiempo de viaje}

El proceso de crear una superficie de tiempo refiere a que lo más importante es contar con las capas adecuadas para correr los diferentes análisis, por ejemplo, la capa de carreteras a nivel nacional es un elemento indispensable para ello, ver figura 11.

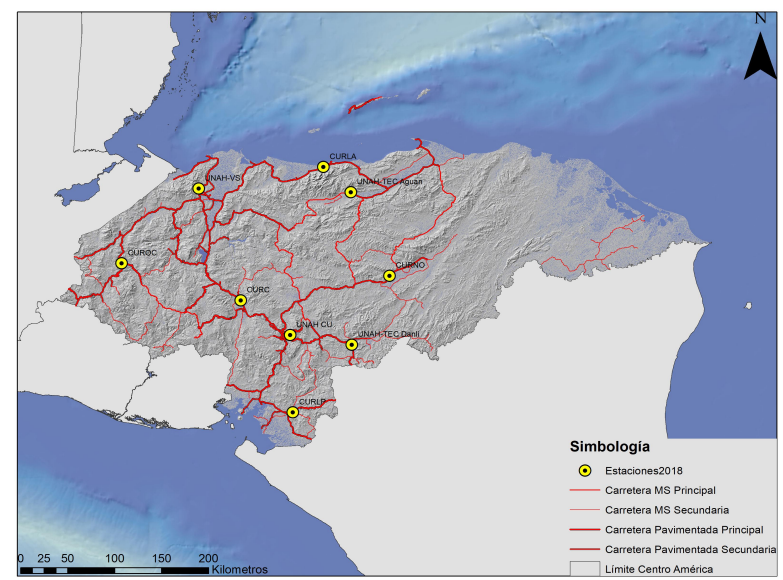

Figura 11: Carreteras que conducen a los Centros Regionales

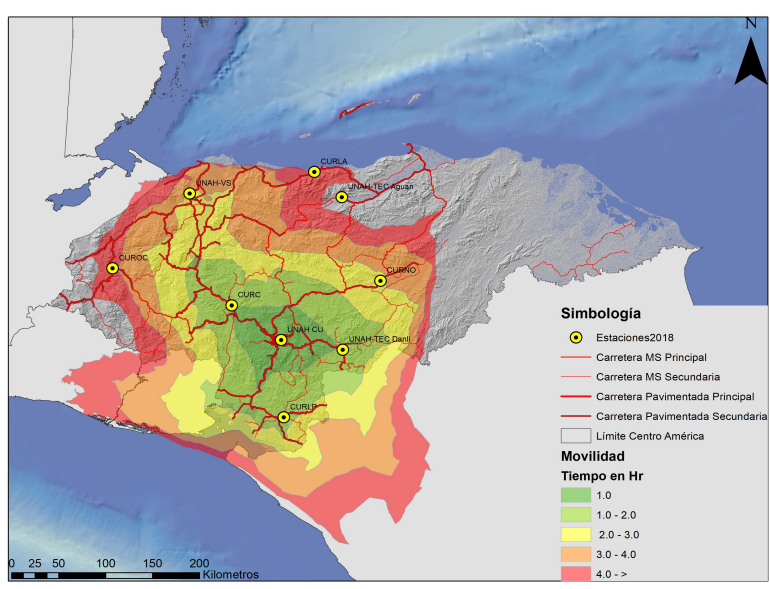

Figura 12: Análisis de tiempo de viaje

Estas capas de información deben de estar proyectadas en el sistema de trabajo que es el WGS84 zona 16 $\mathrm{N}$, información como la distancia de conducción en medio rural el cual está configurado a $5 \mathrm{~km}$ por hora y convertidas a raster ya que las superficies de viaje basadas en raster han demostrado ser herramientas muy poderosas (Hallett y McDermott, 2011). 
Cherkassky et al. (1994) analizan la complejidad de varios algoritmos de ruta más corta para todos y comparan su rendimiento aplicándolos a redes artificiales. Su implementación del algoritmo de Dijkstra con una estructura de datos de doble cubeta tiene el mejor tiempo de cálculo.

El modelo de Dijkstra es un procedimiento reconocido como el mejor de los modelos que resuelve el problema de la trayectoria más corta de origen único en un gráfico ponderado. Este algoritmo es de tipo "greedy" porque en cada iteración elige la mejor opción de las posibles con la esperanza de encontrar así la mejor solución global. Una característica de este algoritmo es la utilización de etiquetas en cada nodo cuya función es indicar en cada iteración del algoritmo la distancia del origen a dicho nodo. En cada iteración una de las etiquetas será "permanente", es decir, indicará la distancia mínima final del nodo inicial a dicho nodo (Revenga, 2008).

El modelo de Dijkstra realiza O(n2) operaciones (sumas y comparaciones) para determinar la longitud del camino más corto entre dos vértices de un grafo ponderado simple, conexo y no dirigido con n vértices.

$$
\text { Tiempo de ejecución }=\mathrm{O}\left(|A| T_{d \kappa}+|v| T_{d m}\right)
$$

Donde:

$|A|$ : Número de aristas

$T_{d \kappa}$ : Complejidad de disminuir clave

$|v|$ : Número de vértices

$T_{d m}$ : Complejidad de extraer mínimo

Crear áreas de tiempo de recorrido por vehículo crea áreas a las que se puede llegar en un tiempo de recorrido por vehículo especificado o que estén a una distancia de conducción dada. Se mide desde uno o varios puntos con la idea de responder la siguiente pregunta: ¿A dónde puedo ir desde aquí conduciendo 60 minutos? Es posible que se encuentre una respuesta a la pregunta solo con visualizar la figura 12.

Las superficies de tiempo de viaje se calcularon utilizando datos ráster, que tienen el potencial de error si no se tratan adecuadamente. Los datos de carreteras se rasterizaron a partir de datos de carreteras vectoriales utilizando el límite de velocidad como un atributo. 
En la tabla 1 se muestra el resultado de determinar la relación de un centro con los demás, estimando los tiempos de viaje sobre la superficie y los vectores calculados.

Tabla 1: Cálculo de tiempo en segundos entre los diferentes centros

\begin{tabular}{|c|c|c|c|c|}
\hline Nombre y destinos & Del punto & Al punto & Nombre del centro regional & Análisis de viaje/ segundos \\
\hline Punto 3:4.0 - 5.0 & 4 & 5 & CUROC & 28598.99301 \\
\hline Punto 3:3.0 - 4.0 & 3 & 4 & CUROC & 20166.58498 \\
\hline Punto 3:2.0 - 3.0 & 2 & 3 & CUROC & 14242.00133 \\
\hline Punto 3:1.0 - 2.0 & 1 & 2 & CUROC & 9245.444546 \\
\hline Punto 3:0 - 1.0 & 0 & 1 & CUROC & 3037.799931 \\
\hline Punto 8:4.0 - 5.0 & 4 & 5 & CURLP & 31719.42087 \\
\hline Punto 8:3.0 - 4.0 & 3 & 4 & CURLP & 27464.82693 \\
\hline Punto 8:2.0 - 3.0 & 2 & 3 & CURLP & 21147.22411 \\
\hline Punto 8:1.0 - 2.0 & 1 & 2 & CURLP & 14287.43986 \\
\hline Punto 8:0 - 1.0 & 0 & 1 & CURLP & 5361.917203 \\
\hline Punto 4:4.0 - 5.0 & 4 & 5 & CURC & 27139.5998 \\
\hline Punto 4:3.0 - 4.0 & 3 & 4 & CURC & 25913.15708 \\
\hline Punto 4:2.0 - 3.0 & 2 & 3 & CURC & 26939.98447 \\
\hline Punto 4:1.0 - 2.0 & 1 & 2 & CURC & 17557.31173 \\
\hline Punto 4:0 - 1.0 & 0 & 1 & CURC & 5758.89151 \\
\hline Punto 9:4.0 - 5.0 & 4 & 5 & UNAH CU & 25932.77373 \\
\hline Punto 9:3.0 - 4.0 & 3 & 4 & UNAH CU & 29309.00874 \\
\hline Punto 9:2.0 - 3.0 & 2 & 3 & UNAH CU & 25095.36432 \\
\hline Punto 9:1.0 - 2.0 & 1 & 2 & UNAH CU & 18645.60022 \\
\hline Punto 9:0 - 1 & 0 & 1 & UNAH CU & 5961.47436 \\
\hline Punto 7:4.0 - 5.0 & 4 & 5 & UNAH-TEC Danlí & 31754.74513 \\
\hline Punto 7:3.0 - 4.0 & 3 & 4 & UNAH-TEC Danlí & 28878.31961 \\
\hline Punto 7:2.0 - 3.0 & 2 & 3 & UNAH-TEC Danlí & 22010.52377 \\
\hline Punto 7:1.0 - 2.0 & 1 & 2 & UNAH-TEC Danlí & 12596.49306 \\
\hline Punto 7:0 - 1 & 0 & 1 & UNAH-TEC Danlí & 5147.283001 \\
\hline Punto 6:4.0 - 5.0 & 4 & 5 & CURNO & 20236.32733 \\
\hline Punto 6:3.0 - 4.0 & 3 & 4 & CURNO & 13193.18809 \\
\hline Punto 6:2.0 - 3.0 & 2 & 3 & CURNO & 10330.94387 \\
\hline Punto 6:1.0 - 2.0 & 1 & 2 & CURNO & 8719.371144 \\
\hline Punto 6:0 - 1.0 & 0 & 1 & CURNO & 4230.65371 \\
\hline Punto 1:4.0 - 5.0 & 4 & 5 & UNAH-VS & 33099.82008 \\
\hline Punto 1:3.0 - 4.0 & 3 & 4 & UNAH-VS & 24216.8877 \\
\hline Punto 1:2.0 - 3.0 & 2 & 3 & UNAH-VS & 18496.44442 \\
\hline Punto 1:1.0 - 2.0 & 1 & 2 & UNAH-VS & 11990.7566 \\
\hline Punto 1:0 - 1 & 0 & 1 & UNAH-VS & 5131.074324 \\
\hline Punto 2:4.0 - 5.0 & 4 & 5 & CURLA & 19973.2247 \\
\hline Punto 2:3.0 - 4.0 & 3 & 4 & CURLA & 16654.58436 \\
\hline
\end{tabular}




\begin{tabular}{lcccc}
\hline Nombre y destinos & Del punto & Al punto & Nombre del centro regional & Análisis de viaje/ segundos \\
\hline Punto 2:2.0 - 3.0 & 2 & 3 & CURLA & 11335.21675 \\
\hline Punto 2:1.0 - 2.0 & 1 & 2 & CURLA & 7047.249758 \\
\hline Punto 2:0 - 1 & 0 & 1 & CURLA & 2998.75667 \\
\hline Punto 5:4.0 - 5.0 & 4 & 5 & UNAH-TEC Aguan & 13440.52524 \\
\hline Punto 5:3.0 - 4.0 & 3 & 4 & UNAH-TEC Aguan & 9901.48697 \\
\hline Punto 5:2.0 - 3.0 & 2 & 3 & UNAH-TEC Aguan & 8660.579801 \\
\hline Punto 5:1.0 - 2.0 & 1 & 2 & UNAH-TEC Aguan & 6754.826123 \\
\hline Punto 5:0 - 1 & 0 & 1 & UNAH-TEC Aguan & 2594.681673 \\
\hline
\end{tabular}

\section{Conclusiones}

Con el objetivo de estar seguros sobre la buena visibilidad satélital y la propia geometría en la región, se caracterizaron diversos análisis con la cobertura espacial del GPS, también se verifico la incertidumbre de la posición (DOP) que también arrojo buenos resultados, en pocas palabras la red de las estaciones permanentes de la UNAH cuenta con una excelente posición GPS, buena disponibilidad de satélites en la región y relativamente no hay obstáculos significativos en cada uno de los centros en donde se encuentran instalados los receptores. Se podría recomendar bajar quizá a $10^{\circ}$ la máscara asumiendo abarcar más observación para cada antena.

Con lo que respecta al tiempo de viaje entre los Centros Regionales, se presentó una metodología para evaluar la eficiencia de los esquemas de tiempo de viaje. La metodología propuesta se aplicó de manera adicional para estimar los tiempos y reducir la demanda del período y seguridad del tiempo de viaje, para ser más eficientes en los cálculos y desempeños a la hora de dar mantenimiento a los equipos instalados en los diferentes centros.

\section{Agradecimientos}

Se agradece a Charles De Mets de la Universidad de Wisconsin y a la Embajada Norteamericana, por su disposición en colaborar con la donación y traslado del equipo GPS ya que sin él no se podrían lograr los diferentes análisis que pueden surgir como subproductos de esta red ya instalada y destacar que es la única red GPS de estaciones activas distribuida a nivel nacional; al equipo colaborador de la Universidad de Alcalá. 


\section{Referencias}

Cherkassky, B. V., Goldberg, A. V., y Radzik, T. (1994). Shortest paths algorithms: Theory and experimental evaluation. En Proceedings of the Fifth Annual ACM-SIAM Symposium on Discrete Algorithms, SODA '94, p. 516-525, USA. Society for Industrial and Applied Mathematics.

Hallett, L. F. y McDermott, D. (2011). Quantifying the extent and cost of food deserts in lawrence, kansas, usa. Applied Geography, 31(4):1210 - 1215. Local Food Systems and the Applied Geography of Food. DOI: https://doi.org/10.1016/j.apgeog.2010.09.006.

Lee, S.-B., Lee, S. M., y Lee, K.-Y. (2019). A Gini coefficient based evaluation on the reliability of travel time forecasting. Journal of King Saud University - Engineering Sciences, 31(4):314 - 319. DOI: https: //doi.org/10.1016/j.jksues.2017.12.001.

Lomax, T., Schrank, D., y Turne, S. (2003). Selecting travel realibity measures. Technical report, Texas Transportation Institute.

Revenga, J. M. A. (2008). Flujo en redes y gestion de proyectos. teoria y ejercicios resueltos. En Flujo en redes y gestion de proyectos. Teoria y ejercicios resueltos.

Sickle, J. V. (2015). GPS for land surveyours. Taylor and Francis Group.

GNSS Agency, E. (2013). GNSS market report. Technical report, GSA European GNSS Agency.

Yahya, M. H. y Kamarudin, m. (2008). Analysis of gps visibility and satellite receiver geometry over different latitudinal regions. En Analysis of GPS Visibility and Satellite Receiver Geometry over Different Latitudinal Regions. 